\title{
Bioinspired Assemblies and Plasmonic Interfaces for Electrochemical Biosensing
}

\author{
Samuel S. Hinman ${ }^{\mathrm{a}}$ and Quan Cheng, ${ }^{\mathrm{a}, \mathrm{,}}$ \\ ${ }^{\mathrm{a}}$ Environmental Toxicology and ${ }^{\mathrm{b}}$ Department of Chemistry, University of California - Riverside, \\ Riverside, CA 92521, USA \\ *Corresponding author. Email: quan.cheng@ucr.edu
}

Submitted to the special issue for the $80^{\text {th }}$ birthday of Professor Hong-Yuan Chen 


\begin{abstract}
Electrochemical biosensing represents a collection of techniques that may be utilized for capture and detection of biomolecules in both simple and complex media. While the instrumentation and technological aspects play important roles in detection capabilities, the interfacial design aspects are of equal importance, and often, those inspired by nature produce the best results. This review highlights recent material designs, recognition schemes, and method developments as they relate to targeted electrochemical analysis for biological systems. This includes the design of electrodes functionalized with peptides, proteins, nucleic acids, and lipid membranes, along with nanoparticle mediated signal amplification mechanisms. The topic of hyphenated surface plasmon resonance assays is also discussed, as this technique may be performed concurrently with complementary and/or confirmatory measurements. Together, smart materials and experimental designs will continue to pave the way for complete biomolecular analyses of complex and technically challenging systems.
\end{abstract}

Keywords: Biosensor, Biochip, Surface functionalization, Lipid membrane, Nanopore, Electrochemical surface plasmon resonance

\title{
1. Introduction
}

Electrochemical methods have continued to be at the forefront of modern bioanalytical sensing due to their sensitivity, ease of use, and low cost, opening up multiple avenues for biomolecular detection to a wide audience. Measurements may be conducted with complex instruments or simple multimeters, making these methods highly accessible. New applications, material designs, and method developments are driving this field forward, which we aim to 
highlight in this Review. The majority of the topics covered are related to recent biochip and/or macroelectrode based detection strategies. These surfaces may be functionalized with a large range of biomolecular signal transducers, of which the compositions, orientations, and underlying material attachments can have profound effects on measurement sensitivity and selectivity. Hyphenated methods with other techniques have also shown interesting advances, such as the combination of surface plasmon resonance and electrochemistry. While both carry their own advantages and disadvantages, the combined results have allowed for complementary and powerful studies to be performed, often concurrently. Though we do not cover the topic of electrochemical analysis of neurotransmitters, we feel this area is of equal importance and recommend the recent reviews by Bucher and Wightman [1] and Nguyen and Venton [2].

\section{Peptide, Protein, and Nucleic Acid Functionalized Interfaces}

Peptide, protein, and nucleic acid based signal transduction have been established as facile and popular routes for biosensing of a variety of molecules in simple and complex media. Generating an electrochemical signal from these interfaces may result from a catalyzed reaction in which a redox reporter is generated, from bringing an immobilized reporter closer to or further away from the working electrode, or from analyte interference with the electron transport path upon application of an electrical potential. These assays may be enhanced through rational design of the biomolecular probes, co-assembly with inorganic materials, and integration of nanomaterials. In this section, we will focus on the interfacial aspects of material design between the above biomolecular signal transducers and their underlying supports.

\subsection{Target Capture with Peptides and Proteins}


The most well known, point of care electrochemical biosensor is the glucometer, which utilizes immobilized enzymes to generate electroactive molecules [3]. Most commercial glucometers rely upon glucose oxidase, which catalyzes the conversion of glucose to hydrogen peroxide, for which a measurable amperometric signal can be detected. While blood glucose has been the medium of choice for glucose testing, tear glucose levels have seen a recent increase in popularity due to their high correlation with blood glucose values and lower invasiveness for sample collection [4-6]. The catalytic properties of immobilized enzymes may also be analyzed directly if the protein has a heme center "wired" to the electrode surface, as Salamifar et al. reported in immobilizing cytochrome $\mathrm{c}$ for hydrogen peroxide detection [7]. In this case, binding of $\mathrm{H}_{2} \mathrm{O}_{2}$ results in concentration dependent change in the cyclic voltammogram for cytochrome c bound at the heme center, which is absent when $\mathrm{H}_{2} \mathrm{O}_{2}$ is exposed without the heme protein.

Antibodies are a popular choice for biomolecular recognition, as their binding affinities are strong and specific, and they may be produced for a wide range of biomolecules. Electrochemical detection of binding events may be achieved in a label-free manner, meaning that neither the analyte nor immobilized recognition unit requires a covalently bound reporter. Protein detection has been accomplished in an arrayed format under this scheme, utilizing nanostructured microelectrodes and antibodies specific for the CA-125 biomarker [8]. Binding of the biomarker to the array results in a blocking of the electron transport path for $\left[\mathrm{Fe}(\mathrm{CN})_{6}\right]^{3-/ 4-}$, present in the flow medium, which can be monitored with differential pulse voltammetry (DPV). This methodology is not only applicable to biomarker proteins, but also toward cell capture and detection. Immobilized anti-EpCAM has been applied toward counting of prostate cancer cells in complex media, and with non-target cells present (Fig. 1) [9]. Circulating tumor cells have also 
been quantified electrochemically in whole blood with signal amplification from an additional detection antibody, and an enzyme-linked reporter [10].

Interesting detection schemes have been realized when linear peptides, instead of larger, folded proteins, are used. One option, if the goal is to capture and/or detect antibodies, is to link a peptide/antigen that the target antibody is specific for to the surface, which has been demonstrated for labeled and direct electrochemical detection of HIV antibodies [11], as well as in a competitive format for screening of the endothelin-1 peptide [12]. Peptide folding based mechanisms may also be utilized, in which one terminus is attached to the working electrode, and the other is modified with a redox label capable of producing an electrochemical response as the labeled terminus folds toward or away from the surface. Anti-p24 was detected under this scheme using a methylene blue terminated peptide and alternating current voltammetry (ACV) [13]. Alternative surface attachment chemistries, including nickel(II)-nitriloacetic acid monolayers for attaching His-tagged peptides [14], and methods to improve peptide sensor specificity through probe alterations [15] are also under investigation. Additionally, peptides may be used concurrently with other recognition elements to function as excellent suppressors of nonspecific binding. Thiolated oligonucleotides are easy to incorporate as diluents in gold sensors $[16,17]$, and the effect of various end terminals on antifouling capabilities has been explored [18].

\subsection{Viral Composite Interfaces}

Incorporation of viruses into sensing materials is a relatively new and biologically inspired approach for binding analytes of interest. The M13 phage in particular makes an excellent and universal bioaffinity platform, as it may be engineered to bind many different types 
of biomolecules [19, 20]. Donavan et al. revealed that electrooxidization of 3,4-

ethylenedioxythiophene (EDOT) in the presence of the M13-K01 phage resulted in a composite polymer/virus film interface that exhibits reasonable sensitivity toward a model polyclonal antibody using electrochemical impedance spectroscopy [21]. Moreover, polymerization of the PEDOT film and incorporation of the virus could be achieved in one step, as opposed to the multi-step conjugation techniques seen in most chip-based studies. Virus concentration within the film may be linearly controlled through increased virus concentration in the monomer solution, which also results in an increase in surface roughness and decrease in composite conductivity [22]. In addition to thin films, PEDOT and M13 may be co-assembled into hybrid nanowires using lithographically patterned nanowire deposition, and this material has been used for biosensing of prostate specific membrane antigen (PSMA) at a concentration of $56 \mathrm{nM}$ [23]. Following genetic encoding of phage ligands, M13 may be further chemically modified, which was performed to obtain two complementary ligands for PSMA on the same phage particle [24]. Electrochemical impedance measurements utilizing this PEDOT/bidentate M13 phage composite exhibited a strong chelating effect for PSMA, extending detection limits to sub-nanomolar levels (Fig. 2).

\subsection{Nucleic Acid Recognition and Folding Strategies}

Nucleic acids can be immobilized and used in many strategies for specific detection of many types of analytes. The most straightforward route is to use unmodified DNA or RNA probes to bind their complimentary sequences. Hierarchical nanostructured electrodes functionalized with thiolated single stranded DNA have been applied toward rapid bacterial mRNA detection [25]. The increase in negative charge from formation of the heteroduplex pair 
influences the electrocatalytic properties of a $\mathrm{Ru}\left(\mathrm{NH}_{3}\right)_{6}{ }^{3+} / \mathrm{Fe}(\mathrm{CN})_{6}{ }^{3-}$ reporter system, which can be monitored using DPV. This sensing system has been applied toward proximal well bacterial cell lysis and mRNA detection for low bacteria concentrations [26], and additionally it has been shown that sensor size, material composition, and probe density influence the analytical sensitivity [27]. Similar sensors have been applied toward identification of circulating tumor cells in prostate cancer patient blood samples [28], analysis of circulating nucleic acids in serum [29], and quantification of biomarkers in donor lungs based on respective mRNA expressions [30]. While gold electrodes are most common for these assays, alternative materials such as silicon are being explored as new and more robust surface attachment chemistries are discovered [31].

Redox-labeled nucleic acid sensors function similarly to redox-labeled peptide sensors, in that folding mechanisms of the strand result in an electrochemical signal as a covalently bound redox reporter moves closer to or further away from the working electrode surface (Fig. 3). Both stem-loop and linear probes sequences may be used for these assays, for which amperometric signals result from the hybridization induced conformational and dynamic changes in the probes, respectively [32]. Multiple signaling reporters on different strands may be integrated into the same experimental design (e.g. methylene blue and ferrocene terminated ssDNA) for confirmation of results $[33,34]$. Conformational changes of the probe may be varied in a facile manner by changing the DNA probe sequence and spacing regions, as signal-off/signal-on methods can take the form of stem-loop formations [32] or deformations [35, 36], respectively. There are a number of factors that may influence the performance of this class of sensors, including the probe conformation/orientation [37, 38], diluent length [38], probe and target flexibility [39], and redox tether length and flexibility [40]. Ion chelating effects of DNA 
heteroduplex pairs have also been shown to affect the probe rigidity, and this aspect has recently been applied toward $\mathrm{Hg}$ (II) mediated glutathione sensing [41], $\mathrm{Ag}(\mathrm{I})$ detection [42], and $\mathrm{Au}(\mathrm{III})$ detection [43].

Aptamers are a class of synthetic nucleic acid sequences that have gone through a systematic selection process (SELEX) for exhibiting a high and specific binding affinity toward the analyte of interest. Aptamers are considerably more stable than many antibodies, and are easy to amplify through polymerase chain reaction. The articles by Song et al. and Cheng et al. provide thorough reviews of aptamer-based biosensors $[44,45]$. One novel advance in electrochemical aptamer based techniques include the neutralizer displacement assay, in which the aptamer target replaces a neutralizing element initially bound to the aptamer probe, resulting in a large change in surface charge [46]. These assays relied on the $\mathrm{Ru}\left(\mathrm{NH}_{3}\right)_{6}{ }^{3+} / \mathrm{Fe}(\mathrm{CN})_{6}{ }^{3-}$ reporter system, monitored through DPV, and were applicable to sensing of DNA, RNA, ATP, thrombin, and cocaine. Folding based aptamers, in which a redox reporter is specifically bound to one part of the aptamer surface for a binding induced electrochemical response, have also been successful for detecting vascular endothelial growth factor in whole blood, and insulin in buffered conditions [47, 48].

\subsection{Metal Nanoparticle Conjugates for Biomolecular Recognition}

Metal nanoparticles have been used in a wide variety of enhancement schemes, owing to their ability to preconcentrate samples out of solution, increase the effective surface area for molecular binding at the working electrode, and possibly contribute to additional electrochemical/catalytic reactions. Aryl diazonium salts may be used to attach gold nanoparticles to glassy carbon electrodes, followed by blocking with carboxylated oligo(ethylene 
glycol) to reduce nonspecific binding $[49,50]$. For detection of HbA1c (a glycosylated hemoglobin), the surface may be modified with a competitor for its corresponding antibody, resulting in a robust sensor that is sensitive and amenable to whole blood analysis [49]. This surface may be further modified with a ferrocene derivative prior to competitor binding for increased sensitivity [50]. Nam et al. also employed gold nanoparticles in a sandwich assay for IgE detection [51]. DNA aptamers were directly attached to gold nanoparticles immobilized on gold electrodes, which bound $\operatorname{IgG}$ from solution, followed by signal amplification through alkaline phosphatase-conjugated anti-IgE. Sensitivities from CV experiments were directly compared with nanoparticle-absent surfaces, where the nanoparticle-modified electrodes displayed superior performance for low concentrations. Screen-printed carbon electrodes have been used to electrodeposit gold nanoparticles when a gold salt precursor is exposed in situ [52, 53]. When covalently modified with tyrosinase, phenol may be enzymatically oxidized, resulting in measurable signals for CV and SWV [52]. Sensitivity of these sensors may be enhanced through consecutive layers of gold nanocubes prior to tyrosinase attachment [53]. While gold nanoparticles are perhaps most popular choice for enhancement, additional shapes and compositions of nanoparticles have been explored, including platinum nanoparticles for DNA hybridization events [54], sintered nanoparticles compatible with paper supports [55], and a combination of copper and silver nanoparticles for microsome and exosome detection [56]. In addition to capturing analytes directly at the working electrode surface, nanoparticles may be dispersed for capture of analytes in solution, followed by magnetic pull-down onto the working electrode. This allows for ultrasensitive biosensing of low analyte concentrations in a manner that is much faster than Langmuir adsorption (i.e. waiting for the analyte to diffuse to the surface). Silver nanoparticles have been used to label model analytes in solution, which were 
then conjugated with magnetic microbeads through biotin-streptavidin interactions. These conjugates were pulled down to a paper analytical device, with 250,000-fold amplification for model analytes over conventional sensing methods [57]. The silver nanoparticle system was later applied toward detection of hepatitis B viral DNA, with a detection limit of $85 \mathrm{pM} \mathrm{[58].} \mathrm{Chuah}$ et al. produced gold nanoparticles with magnetic cores, on which a sandwich ELISA for prostate specific antigen could be conducted in solution, followed by magnetic pull-down to the planar electrode surface [59]. The faradaic electrochemistry of this scheme could be switched on and off through application of passivating self-assembled monolayers on the working electrode [60]. Recently, a number of shapes and sizes of gold-coated magnetic nanoparticles were compared for this assay, with cubic nanoparticles of $c a .50 \mathrm{~nm}$ diameter presenting the best stability and performance over spherical and raspberry morphologies [61].

\section{Lipid Membranes and Nanopore Signal Transduction}

The lipid membrane represents a fundamental protective structure for communication and material exchange in biological systems. While the plasma membrane environment is a naturally complex interface, a number of efforts have been made to simplify it into solid supported structures over the past couple of decades [62]. These have collectively allowed for biophysical studies of embedded biomolecules and membrane interactions, in addition to exploitation of the interface for biologically inspired sensors. Utilizing model lipid systems carries the advantage of being able to alter and tune the composition and constituents of the membrane, so that targeted biomolecular assays may be carried out, and if desired, with tailored biophysical properties (e.g. membrane stability and lipid fluidity) [63]. There are many routes that may be taken to create a supported membrane structure for electrochemical assays, which include: 1) tethering 
macromolecular lipid structures to a modified electrode (e.g. bilayer vesicles, micelles, other self-assembled nano/microstructures); 2) fusing vesicles into a planar membrane supported by an underlying surface (i.e. working electrode); or 3) suspending a bilayer membrane over a narrow aperture, which allows for direct measurement of current flow through membrane channels (Fig. 4).

\subsection{Surface-Bound Membrane Structures}

Due to their amphiphilic nature, most phospholipids will spontaneously self-assemble into spherical structures in solution, which can be further shaped and sized using established preparatory techniques [64]. Direct conjugation of these functional structures to a support without further self-assembly schemes is an attractive avenue for decreasing sensor fabrication and preparation time. The incorporation of surface binding ligands into small, unilamellar vesicles (SUVs) for amperometric characterization of streptolysin O (SLO) was demonstrated by $\mathrm{Xu}$ and Cheng [65]. SUVs assembled from phosphatidylcholine, cholesterol, diacetyl phosphate, and 1-octadecanethiol were formed in a buffer containing the redox probe $\mathrm{K}_{3} \mathrm{Fe}(\mathrm{CN})_{6}$, which was encapsulated during the vesicle formation process. The 1-octadecanethiol constituent was evenly distributed with the other lipids, and allowed for direct attachment of the vesicles to gold disk electrodes. Upon introduction of the pore-forming toxin SLO, the redox probe was released, resulting in a quantifiable change in the amperometric signal. Cheng et al. later applied similar methods for anchoring of polydiacetylene (PDA) lipid microstructures and voltammetric sensing of cholera toxin (CT) [66]. The platelet lipid assemblies were incorporated with ferrocene and cysteine conjugated PDAs for electrochemical activity and gold surface attachment, respectively. With cell surface ligand $\mathrm{GM}_{1}$ co-assembled, these structures were capable of recognizing $\mathrm{CT}$ and 
producing a voltammetric response through blocking of the electron transport path, thus decreasing Faradaic current. Recently, transmembrane proteins reconstituted into liposomes have been studied through ex situ voltammetry and chronopotentiometry [67]. This method relies on the aspect that the proteins used were electroactive compared to the lipid bilayer, and allowed for studies of $\mathrm{Na}^{+} / \mathrm{K}^{+}$-ATPase and mitochondrial uncoupling protein 1 in their native folded states. Other artificial lipid assemblies, including protein scaffold constructs (i.e. nanodiscs) [68] and PEG-stablized lipodisks [69], may be capable of similar measurements described above, though to the best of our knowledge, have not yet been interfaced with electrochemical techniques.

Surface wettability plays an important role in further self-assembly processes of lipid vesicles. When exposed to a surface that is highly hydrophobic, free lipids or SUVs will fuse into a planar hybrid bilayer membrane (HBM), where the upper leaflet of the membrane is composed of phospholipids and the lower leaflet is a derivitized surface (often, a hydrophobic SAM) [70]. Micropatterned HBMs have been used to quantify SLO on alkanethiol substrates through voltammetric responses of ferrocene carboxylic acid (FCA) [71]. Formation of the HBM prevents access of FCA to the electrode surface, while pore formation by SLO restores redox response. This system was also found to be compatible with hexyl thioctate and thioctic acid tri(ethylene glycol) ester modified gold electrodes, which are capable of supporting HBMs and allow for more sensitive characterizations of pore forming toxins $[72,73]$. Hydrophobic SAM modified electrodes covalently bound to a $\mathrm{Cu}(\mathrm{I}) / \mathrm{Cu}$ (II) redox center have recently been used to characterize anion transport through hybrid bilayer membranes, and show the transport to primarily follow a solubility-diffusion mechanism, rather than a pore mechanism [74]. In an alternative HBM approach, Ma et al. developed a gold-attached SAM, terminated with an ubiquinone moiety, which was capable of tethering a lipid bilayer from the surface $[75,76]$. 
Ubiquinone facilitated redox coupling between embedded $\mathrm{NADH} / \mathrm{NAD}^{+}$, which was monitored through cyclic voltammetry and surface-enhanced Raman spectroscopy (Fig. 5). More recent efforts have been made to preserve membrane structure during storage and transport, which may have important implications toward advancing commercialization and widespread adaptation of these biomimetic interfaces [77, 78].

\subsection{Black Lipid Membranes with Embedded Nanopores}

Black lipid membranes (BLMs) are lipid bilayers that have been formed over a narrow aperture (dia. $<1 \mathrm{~mm}$ ), separating two distinct aqueous regions [62, 79]. BLMs are typically supported at their periphery by a hydrophobic support, which anchors the membrane in place through hydrophobic interactions with the phospholipid tails. Electrodes may then be placed on either side of the membrane for measurements of current, often accompanied by sensing across embedded channel proteins. While carrying the advantage of high transmembrane protein mobility, a feature often lacking in HBMs, BLMs are inherently less stable and many recent efforts have been made to improve the structural robustness of this class of materials. Polymerization of adjacent modified phospholipids is one popular option for improving stability, and has been tested using dienoyl- and sorbyl-modified phospholipids [80]. Each of the photopolymerized membranes exhibited significantly higher stability after UV exposure compared to diphytanoyl phosphocholine (DPhPC), extending the lifetime of these structures from hours to days. Methacrylate polymer precursors may be partitioned into DPhPC BLMs to create polymer scaffolds that enhance the stability in a more cost effective and accessible manner [81]. Surface energy of the hydrophobic support also has an impact on the electrical, mechanical, and stability properties, and can be altered through use of different hydrophobic monolayer 
coatings [82]. In addition to glass apertures coated with various monolayers, BLMs can be formed over polymer substrates (e.g. SU-8), for which the geometry can be precisely tuned and defined using standard photolithography techniques [83]. For further information on emerging microaperture fabrication techniques for BLM studies has recently been highlighted by Baker and Aspinwall [84].

Nanopore sensing is an exciting concept derived from biomimetic BLMs. Biological nanopores are typically constructed from transmembrane pore-forming proteins, which result in pores with diameters of 10-30 $\AA$ that may be used for measuring the translocation of biomolecules based on their size or aggregation state [85]. $\alpha$-Hemolysin $(\alpha-\mathrm{HL})$ is the most commonly used protein for these analyses, though alternative proteins such as aerolysin and SP1 are also being explored with promising results for peptide and DNA detection [86-88]. Biosensing is accomplished through measurements of current across the membrane, where binding or translocation events result in measurable step reductions in the presence of an applied potential. Accurate data processing is often crucial toward filtering electronic noise while discerning pore blockage dwell times and step magnitudes, which are indicative of binding affinity and molecular weight, respectively [89]. A plethora of bulk and single molecule applications exist for nanopore mediated biosensing, from peptide and protein interactions to nucleic acid sequencing [90]. Wang et al. applied the $\alpha$-HL nanopore toward analysis of $\beta$ amyloid aggregation states in the presence of $\beta$-cyclodextrin, which has implications for the development and treatment of Alzheimer's disease [91]. The different aggregation states and protofilament formations could be monitored through characteristic step currents, which showed whether the macromolecular complexes were bumping into the pore or translocating through due to their shape and/or size. This experimental setup was also used to monitor states of $\alpha$-synuclein 
fibrillation, which may function as a biomarker of Parkinson's disease [92]. In addition to proteins, aptamer conformational changes may be monitored through $\alpha$-HL nanopores, and an ATP-binding aptamer in its folded, ATP-bound, and linear conformations were distinguished [93]. Interactions between nucleic acids and other biomolecules have also been of high interest, and nanopore studies have shown that antibody binding enhances the translocation of poly $(\mathrm{dT})_{45}$ through $\alpha$-HL, compared to translocation of poly $(\mathrm{dT})_{45}$ alone [94]. Recently, Zhang et al. applied $\alpha$-HL to study light-regulated interactions between spiropyran, a photochromic molecule, and an RNA binding aptamer, which was capable of having its translocation being turned on or off by the photoisomerization of bound spiropyran [95].

\section{Hyphenated Assays with Surface Plasmon Resonance}

In this section, an overview of planar (conventional) surface plasmon resonance (SPR) measurements coupled to electrochemical techniques will be discussed (EC-SPR), though the topic of nanostructured SPR systems with electrochemical applications is also of wide interest and has been reviewed [96]. SPR is a label-free optical technique for measuring adsorption and immobilization events in real-time at a suitable metal interface (e.g. Au, $\mathrm{Ag}, \mathrm{Cu}, \mathrm{Pt})$. This phenomenon occurs under conditions specific to the material, in which light is able to couple with the free conduction electrons via a momentum transfer, resulting in an oscillating electromagnetic field (in the form of surface plasmon polaritons, SPPs) that propagates parallel to the surface of the metal film and exponentially decays with perpendicular distance. The resonance conditions are governed by a number of properties, which for the purpose of biosensing include the wavelength of incident light $(\lambda)$, the angle of incidence $\left(\theta_{0}\right)$, and the dielectric constant of the surrounding medium. Both $\lambda$ and $\theta_{0}$ are dependent on the SPR 
instrument and material design, while the dielectric constant is affected by binding events and bulk refractive index changes at the sensor surface [97]. For a more comprehensive survey of modern SPR theory, techniques, and instrumentation, the perspective by Couture et al. provides an excellent overview of these topics [98]. The label-free nature of SPR measurements affords minimized sample preparation, and when combined with signal amplification strategies may reach subattomolar limits of detection [99]. The most common experimental setup utilizes an incident light source, coupling prism coated with a thin gold film $(c a .50 \mathrm{~nm})$, and detector arranged in reflectance geometry. The open architecture of many commercial SPR instruments allows for the incorporation of electrochemical equipment that either uses the gold film of the SPR sensor chip as the working electrode, or introduces additional working electrodes for orthogonal sensing in the nearby flow medium (Fig. 6). This combined methodology carries a number of advantages, including the ability to obtain multidimensional data from the same system (i.e. biophysical and electrochemical), as well as the confirmation of data from an additional sensing modality to give higher confidence to reported results.

\subsection{Experimental Considerations of EC-SPR}

The interaction between applied electric fields and SPPs is quite interesting, and requires special considerations for the methods to be used simultaneously and effectively. The simple process of charging the gold surface above the potential of zero charge has been shown to produce an SPR response, as the complex dielectric constants of gold are potential dependent; however, this effect may be effectively suppressed by densely packed monolayers on the gold surface [100]. Early work in this area involved performing cyclic voltammetry on goldimmobilized redox protein monolayers while observing corresponding SPR responses in an in 
situ fashion [101, 102]. The SPR angular shifts were found to coincide with the ramping potential used for $\mathrm{CV}$, and could be visualized directly or by taking the time derivative of the response [101]. In tuning the incident light wavelength closer to, or farther away from, the absorbance maximum of the protein of interest, conformational changes and information regarding the electronic states during CV can also be distinguished [102]. Wang et al. reasoned that EC-SPR could be used to directly measure convolution voltammetry, which is proportional to the concentration of the diffusing electroactive analyte, without having to numerically compute a convolution integral of faradaic current as seen in traditional analyses of voltammetric currents and peaks [103]. This principle was later applied to EC-SPR measurements of diffusioncontrolled fully reversible, quasi-reversible, and irreversible redox reactions in model systems [104].

\subsection{EC/EIS-SPR Enhanced Biosensing}

SPR has been used to visualize electrochemical processes in a number of biomolecular systems, and to optimize new electroactive materials for biosensing applications [105]. Potentials applied to monolayer nucleic acid films on gold allowed for SPR studies of electric field-induced hybridization and electrostatic denaturation of DNA duplexes. The DNA probes in these studies

remained stable at the potentials used $\left(10^{9} \mathrm{~V} / \mathrm{s}\right)$, and label-free discrimination of base mismatches was achieved [100]. A similar experimental setup was used to monitor target DNA recognition using SPR and ACV concurrently, which allowed for optimal nucleic acid probe sequence selection from the optical and electrochemical data [106]. Cross-platform protein recognition, important in the field of immunosorbent assays (ELISA), has been successful via a "membrane cloaking" method that was developed for complementary results to be obtained by SPR and 
amperometric techniques [107]. Once antibodies were immobilized over a self-assembled monolayer, a cloaking phospholipid membrane was applied to the surface, which was resistant to nonspecific binding and could be removed once the assay was complete. CV and DPV were used to optimize the biochip, while SPR and amperometry were utilized for detection and quantification of $\mathrm{IgG}$ in serum.

Electrochemical impedance spectroscopy (EIS) in combination with SPR (EIS-SPR) is one combination of methods with unique implications for sensing, as the non-invasive nature of EIS allows for measurements through semi-conducting layers and potential coupling with plasmonic response. Gold thin films have been used for EIS-SPR measurements, in addition interdigitated gold electrodes that function as optical diffraction gratings for SPR [108]. The combination was first utilized to monitor DNA hybridization on nanoscale $\mathrm{SiO}_{\mathrm{x}}$ layers on gold, with SPR providing confirmational data to that obtained from EIS studies [109]. Building on this idea, one can conduct plasmonic-based EIS (P-EIS) on an AC-applied gold film, in which the electrochemical impedance and conventional SPR response are simultaneously optically imaged through the AC and DC response components, respectively (Fig. 7) [110]. P-EIS was found to be less sensitive to bulk refractive index changes and non-specific binding compared to conventional SPR assays. Furthermore, the concept was applied to multiplexed array imaging, where the AC SPR phase component easily distinguished between various charge states in small $(<100 \mathrm{Da})$ molecules, and monitored their binding kinetics to arrayed target proteins [111, 112].

\subsection{Hyphenated Assays and Complex Media}

Complex systems (e.g. mammalian cells, bacteria, serum, blood) represent a difficult goal in the biosensing community, and designing assays that remain specifically targeted for multiple 
detection modalities is an even greater task. DNA stem-loop probes that produce responses through folding or unfolding have proven to be effective for sensing of oligonucleotides specific to drug-resistant tuberculosis and Escherichia coli in whole blood [113]. Zhang et al. utilized an immobilization scheme for bacterial studies, in which a monolayer of poly(Arg-Gly-Asp) peptides was self-assembled on the gold film and exposed to a suspension of $E$. coli cells. The cells remained attached to the modified substrate and were characterized by SPR, CV, and EIS. Herring sperm DNA (hsDNA) was exposed to the immobilized bacteria, which was shown to adsorb to $E$. coli cell surfaces with kinetic parameters that were in agreement with those from other studies [114]. Wu et al. recently applied an alternative approach toward the study of live cancer cells by SPR and CV, where HepG2 cells were cultured directly on gold sensor chips prior to insertion into the SPR instrument [115]. When treated with danorubicin, notable morphology and mass changes led to shifts in the SPR signal, while in situ CV was used to monitor danorubicin uptake from the culture medium. Drug uptake was confirmed using traditional microscopy assays, and decreases in the SPR signal were directly correlated with cell survival rates, providing a complementary method for assessing chemotherapeutic effectiveness and cytotoxicity (Fig. 8).

\section{Perspective}

Despite the length of time it has been established, the field of electrochemical biosensing continues to grow as a product of better understandings regarding biomolecular interactions and the introduction of new signal transduction strategies. Supramolecular assemblies and other biomolecular interfaces can be now precisely controlled to tailor functional groups at a new level. Additionally, advances in nanomaterials have provided fresh opportunities to enhance 
electrochemical signal acquisition toward varying degrees of amplification. Continuing challenges that remain include adapting these interfaces toward new biomolecular targets, along with handling samples containing ultra-low analyte concentrations. Beyond antibodies, the adaptation of non-conventional platforms (e.g. nucleic acids/aptamers, phages, and biomimetic membranes) may assist in target recognition as part of the growing biomolecular toolkit used for sensor design. While electrochemical signal transduction through these platforms is capable of achieving ultrasensitive measurements, even down to the single molecule level, the process of Langmuir adsorption to the electrode surface limits real-world applications as respectively longer incubation times are needed for binding. Dispersible electrodes and magnetic pull-down assays have offered novel solutions to this issue, though further improvement will be realized through integration with microfluidic platforms and electrochemically based sample handling strategies [116]. In addition to the handling techniques, integrating complementary sensing modalities will offer further insight into surface structures for improved electron transfer and electrochemical signaling mechanisms. The wide use of compatible surface techniques such as SPR and QCM facilitates the characterization of surface interactions in a label-free manner, which is not always possible for molecules that require redox reporters in amperometry. Additional surface-based optical and mass spectrometric measurements will undoubtedly elucidate these processes toward more complete understandings as the integrations become more timesaving and efficient $[117$, 118]. Taken together, these developments and opportunities are indicative of a bright future for the field.

\section{Abbreviations}


$\alpha$-HL, $\alpha$-hemolysin; ACV, alternating current voltammetry; BLM, black lipid membrane; CT, cholera toxin; CV, cyclic voltammetry; DPV, differential pulse voltammetry; EC-SPR, electrochemical-surface plasmon resonance; EIS, electrochemical impedance spectroscopy; ELISA, enzyme-linked immunosorbent assay; HBM, hybrid bilayer membrane; PDA, polydiacetylene; PEDOT, poly(3,4-ethylenedioxythiophene); QCM, quartz crystal microbalance; SAM, self-assembled monolayer; SLO, streptolysin O; SUV, small unilamellar vesicle; SPP, surface plasmon polariton; SPR, surface plasmon resonance; SWV, square wave voltammetry

\section{Acknowledgements}

We acknowledge financial support from the National Science Foundation (CHE-1413449).

S.S.H. was supported by an NIEHS T32 Training Grant (T32 ES018827). 


\section{References}

[1] E.S. Bucher, R.M. Wightman, Electrochemical analysis of neurotransmitters, Annu. Rev. Anal. Chem. 8 (2015) 239-261.

[2] M.D. Nguyen, B.J. Venton, Fast-scan cyclic voltammetry for the characterization of rapid adenosine release, Comput. Struct. Biotechnol. J. 13 (2015) 47-54.

[3] N.S. Oliver, C. Toumazou, A.E. Cass, D.G. Johnston, Glucose sensors: A review of current and emerging technology, Diabetic Med. 26(3) (2009) 197-210.

[4] Q.Y. Yan, B. Peng, G. Su, B.E. Cohan, T.C. Major, M.E. Meyerhoff, Measurement of tear glucose levels with amperometric glucose biosensor/capillary tube configuration, Anal. Chem. 83(21) (2011) 8341-8346.

[5] B. Peng, J. Lu, A.S. Balijepalli, T.C. Major, B.E. Cohan, M.E. Meyerhoff, Evaluation of enzyme-based tear glucose electrochemical sensors over a wide range of blood glucose concentrations, Biosens. Bioelectron. 49 (2013) 204-209.

[6] K.H. Cha, G.C. Jensen, A.S. Balijepalli, B.E. Cohan, M.E. Meyerhoff, Evaluation of commercial glucometer test strips for potential measurement of glucose in tears, Anal. Chem. 86(3) (2014) 1902-1908.

[7] S.E. Salamifar, S. Lee, R.Y. Lai, Electrochemical hydrogen peroxide sensors fabricated using cytochrome c immobilized on macroelectrodes and ultramicroelectrodes, Colloids Surf., B 123 (2014) 866-869.

[8] J. Das, S.O. Kelley, Protein detection using arrayed microsensor chips: Tuning sensor footprint to achieve ultrasensitive readout of CA-125 in serum and whole blood, Anal. Chem. 83(4) (2011) 1167-1172.

[9] M. Moscovici, A. Bhimji, S.O. Kelley, Rapid and specific electrochemical detection of prostate cancer cells using an aperture sensor array, Lab Chip 13(5) (2013) 940-946.

[10] T.S. Safaei, R.M. Mohamadi, E.H. Sargent, S.O. Kelley, In situ electrochemical ELISA for specific identification of captured cancer cells, ACS Appl. Mater. Interfaces 7(26) (2015) 1416514169.

[11] A. Bhimji, A.A. Zaragoza, L.S. Live, S.O. Kelley, Electrochemical enzyme-linked immunosorbent assay featuring proximal reagent generation: Detection of human immunodeficiency virus antibodies in clinical samples, Anal. Chem. 85(14) (2013) 6813-6819. 
[12] A.T. Sage, X.H. Bai, M. Cypel, M.Y. Liu, S. Keshavjee, S.O. Kelley, Using the inherent chemistry of the endothelin-1 peptide to develop a rapid assay for pre-transplant donor lung assessment, Analyst 140(24) (2015) 8092-8096.

[13] J.Y. Gerasimov, R.Y. Lai, Design and characterization of an electrochemical peptide-based sensor fabricated via "click" chemistry, Chem. Commun. 47(30) (2011) 8688-8690.

[14] A.J. Zaitouna, R.Y. Lai, An electrochemical peptide-based ara h 2 antibody sensor fabricated on a nickel(ii)-nitriloacetic acid self-assembled monolayer using a his-tagged peptide, Anal. Chim. Acta 828 (2014) 85-91.

[15] A.J. Zaitouna, A.J. Maben, R.Y. Lai, Incorporation of extra amino acids in peptide recognition probe to improve specificity and selectivity of an electrochemical peptide-based sensor, Anal. Chim. Acta 886 (2015) 157-164.

[16] O.R. Bolduc, J.N. Pelletier, J.F. Masson, SPR biosensing in crude serum using ultralow fouling binary patterned peptide sam, Anal. Chem. 82(9) (2010) 3699-3706.

[17] A. McQuistan, A.J. Zaitouna, E. Echeverria, R.Y. Lai, Use of thiolated oligonucleotides as anti-fouling diluents in electrochemical peptide-based sensors, Chem. Commun. 50(36) (2014) 4690-4692.

[18] A.J. Zaitouna, J. Joyce, R.L. Cerny, P.H. Dussault, R.Y. Lai, Comparison of mannose, ethylene glycol, and methoxy-terminated diluents on specificity and selectivity of electrochemical peptide-based sensors, Anal. Chem. 87(13) (2015) 6966-6973.

[19] V. Nanduri, I.B. Sorokulova, A.M. Samoylov, A.L. Simonian, V.A. Petrenko, V. Vodyanoy, Phage as a molecular recognition element in biosensors immobilized by physical adsorption, Biosens. Bioelectron. 22(6) (2007) 986-992.

[20] R. Peltomaa, I. Lopez-Perolio, E. Benito-Pena, R. Barderas, M.C. Moreno-Bondi, Application of bacteriophages in sensor development, Anal. Bioanal. Chem. 408(7) (2016) 18051828.

[21] K.C. Donavan, J.A. Arter, R. Pilolli, N. Cioffi, G.A. Weiss, R.M. Penner, Virus-poly(3,4ethylenedioxythiophene) composite films for impedance-based biosensing, Anal. Chem. 83(7) (2011) 2420-2424.

[22] K.C. Donavan, J.A. Arter, G.A. Weiss, R.M. Penner, Virus-poly(3,4ethylenedioxythiophene) biocomposite films, Langmuir 28(34) (2012) 12581-12587. 
[23] J.A. Arter, J.E. Diaz, K.C. Donavan, T. Yuan, R.M. Penner, G.A. Weiss, Virus-polymer hybrid nanowires tailored to detect prostate-specific membrane antigen, Anal. Chem. 84(6) (2012) 2776-2783.

[24] K. Mohan, K.C. Donavan, J.A. Arter, R.M. Penner, G.A. Weiss, Sub-nanomolar detection of prostate-specific membrane antigen in synthetic urine by synergistic, dual-ligand phage, J. Am. Chem. Soc. 135(20) (2013) 7761-7767.

[25] L. Soleymani, Z.C. Fang, B. Lam, X.M. Bin, E. Vasilyeva, A.J. Ross, E.H. Sargent, S.O. Kelley, Hierarchical nanotextured microelectrodes overcome the molecular transport barrier to achieve rapid, direct bacterial detection, ACS Nano 5(4) (2011) 3360-3366.

[26] J.D. Besant, J. Das, E.H. Sargent, S.O. Kelley, Proximal bacterial lysis and detection in nanoliter wells using electrochemistry, ACS Nano 7(9) (2013) 8183-8189.

[27] J. Das, S.O. Kelley, Tuning the bacterial detection sensitivity of nanostructured microelectrodes, Anal. Chem. 85(15) (2013) 7333-7338.

[28] I. Ivanov, J. Stojcic, A. Stanimirovic, E. Sargent, R.K. Nam, S.O. Kelley, Chip-based nanostructured sensors enable accurate identification and classification of circulating tumor cells in prostate cancer patient blood samples, Anal. Chem. 85(1) (2013) 398-403.

[29] J. Das, I. Ivanov, L. Montermini, J. Rak, E.H. Sargent, S.O. Kelley, An electrochemical clamp assay for direct, rapid analysis of circulating nucleic acids in serum, Nat Chem 7(7) (2015) 569-75.

[30] A.T. Sage, J.D. Besant, L. Mahmoudian, M. Poudineh, X. Bai, R. Zamel, M. Hsin, E.H. Sargent, M. Cypel, M. Liu, S. Keshavjee, S.O. Kelley, Fractal circuit sensors enable rapid quantification of biomarkers for donor lung assessment for transplantation, Sci. Adv. 1(7) (2015) e1500417.

[31] P. Michaels, M.T. Alam, S. Ciampi, W. Rouesnel, S.G. Parker, M.H. Choudhury, J.J. Gooding, A robust DNA interface on a silicon electrode, Chem. Commun. 50(58) (2014) 78787880.

[32] W.W. Yang, R.Y. Lai, Comparison of the stem-loop and linear probe-based electrochemical DNA sensors by alternating current voltammetry and cyclic voltammetry, Langmuir 27(23) (2011) 14669-14677. 
[33] W.W. Yang, R.Y. Lai, Integration of two different sensing modes in an electrochemical DNA sensor for approximation of target mismatch location, Electrochem. Commun. 13(9) (2011) 989-992.

[34] W.W. Yang, R.Y. Lai, A dual-signalling electrochemical DNA sensor based on target hybridization-induced change in DNA probe flexibility, Chem. Commun. 48(69) (2012) 87038705.

[35] Z.G. Yu, R.Y. Lai, A reagentless and reusable electrochemical DNA sensor based on target hybridization-induced stem-loop probe formation, Chem. Commun. 48(85) (2012) 10523-10525. [36] Y. Wu, R.Y. Lai, Development of a "signal-on" electrochemical DNA sensor with an oligothymine spacer for point mutation detection, Chem. Commun. 49(33) (2013) 3422-3424. [37] Z.G. Yu, R.Y. Lai, Effect of signaling probe conformation on sensor performance of a displacement-based electrochemical DNA sensor, Anal. Chem. 85(6) (2013) 3340-3346. [38] R. Tavallaie, N. Darwish, M. Gebala, D.B. Hibbert, J.J. Gooding, The effect of interfacial design on the electrochemical detection of DNA and microrna using methylene blue at lowdensity DNA films, Chemelectrochem 1(1) (2014) 165-171.

[39] Y. Wu, R.Y. Lai, Effects of DNA probe and target flexibility on the performance of a "signal-on" electrochemical DNA sensor, Anal. Chem. 86(17) (2014) 8888-8895.

[40] Z.G. Yu, A.J. Zaitouna, R.Y. Lai, Effect of redox label tether length and flexibility on sensor performance of displacement-based electrochemical DNA sensors, Anal. Chim. Acta 812 (2014) 176-183.

[41] H.R.L.Z. Zhad, R.Y. Lai, A Hg(II)-mediated "signal-on" electrochemical glutathione sensor, Chem. Commun. 50(61) (2014) 8385-8387.

[42] Y. Wu, R.Y. Lai, A reagentless DNA-based electrochemical silver(I) sensor for real time detection of $\operatorname{Ag}(\mathrm{I})$ - the effect of probe sequence and orientation on sensor response, Biotechnol. J. (2016).

[43] Y. Wu, R.Y. Lai, Electrochemical gold(III) sensor with high sensitivity and tunable dynamic range, Anal. Chem. 88(4) (2016) 2227-2233.

[44] S.P. Song, L.H. Wang, J. Li, J.L. Zhao, C.H. Fan, Aptamer-based biosensors, TrAC, Trends Anal. Chem. 27(2) (2008) 108-117.

[45] A.K.H. Cheng, D. Sen, H.Z. Yu, Design and testing of aptamer-based electrochemical biosensors for proteins and small molecules, Bioelectrochemistry 77(1) (2009) 1-12. 
[46] J. Das, K.B. Cederquist, A.A. Zaragoza, P.E. Lee, E.H. Sargent, S.O. Kelley, An

ultrasensitive universal detector based on neutralizer displacement, Nat. Chem. 4(8) (2012) 642648.

[47] S. Zhao, W.W. Yang, R.Y. Lai, A folding-based electrochemical aptasensor for detection of vascular endothelial growth factor in human whole blood, Biosens. Bioelectron. 26(5) (2011) 2442-2447.

[48] J.Y. Gerasimov, C.S. Schaefer, W.W. Yang, R.L. Grout, R.Y. Lai, Development of an electrochemical insulin sensor based on the insulin-linked polymorphic region, Biosens. Bioelectron. 42 (2013) 62-68.

[49] G.Z. Liu, S.G. Iyengar, J.J. Gooding, An electrochemical impedance immunosensor based on gold nanoparticle-modified electrodes for the detection of HbA1c in human blood, Electroanalysis 24(7) (2012) 1509-1516.

[50] G.Z. Liu, S.G. Iyengar, J.J. Gooding, An amperometric immunosensor based on a gold nanoparticle-diazonium salt modified sensing interface for the detection of HbA1c in human blood, Electroanalysis 25(4) (2013) 881-887.

[51] E.J. Nam, E.J. Kim, A.W. Wark, S. Rho, H. Kim, H.J. Lee, Highly sensitive electrochemical detection of proteins using aptamer-coated gold nanoparticles and surface enzyme reactions, Analyst 137(9) (2012) 2011-2016.

[52] M.N. Karim, H.J. Lee, Amperometric phenol biosensor based on covalent immobilization of tyrosinase on Au nanoparticle modified screen printed carbon electrodes, Talanta 116 (2013) 991-996.

[53] M.N. Karim, J.E. Lee, H.J. Lee, Amperometric detection of catechol using tyrosinase modified electrodes enhanced by the layer-by-layer assembly of gold nanocubes and polyelectrolytes, Biosens. Bioelectron. 61 (2014) 147-151.

[54] T.M. Alligrant, E.G. Nettleton, R.M. Crooks, Electrochemical detection of individual DNA hybridization events, Lab Chip 13(3) (2013) 349-354.

[55] D.D. Liana, B. Raguse, L. Wieczorek, G.R. Baxter, K. Chuah, J.J. Gooding, E. Chow, Sintered gold nanoparticles as an electrode material for paper-based electrochemical sensors, RSC Adv. 3(23) (2013) 8683-8691. 
[56] Y.G. Zhou, R.M. Mohamadi, M. Poudineh, L. Kermanshah, S. Ahmed, T.S. Safaei, J. Stojcic, R.K. Nam, E.H. Sargent, S.O. Kelley, Interrogating circulating microsomes and exosomes using metal nanoparticles, Small 12(6) (2016) 727-732.

[57] K. Scida, J.C. Cunningham, C. Renault, I. Richards, R.M. Crooks, Simple, sensitive, and quantitative electrochemical detection method for paper analytical devices, Anal. Chem. 86(13) (2014) 6501-6507.

[58] X. Li, K. Scida, R.M. Crooks, Detection of hepatitis B virus DNA with a paper electrochemical sensor, Anal. Chem. 87(17) (2015) 9009-9015.

[59] K. Chuah, L.M.H. Lai, I.Y. Goon, S.G. Parker, R. Amal, J.J. Gooding, Ultrasensitive electrochemical detection of prostate-specific antigen (PSA) using gold-coated magnetic nanoparticles as 'dispersible electrodes', Chem. Commun. 48(29) (2012) 3503-3505.

[60] L. Carter, K. Chuah, R. Tavallaie, A. Barfidokht, S.G. Parker, J.J. Gooding, Switching "on and off" faradaic electrochemistry at an otherwise passivated electrode using gold-coated magnetic nanoparticles, Electrochem. Commun. 61 (2015) 93-96.

[61] S. Moraes Silva, R. Tavallaie, M. Tanzirul Alam, K. Chuah, J.J. Gooding, A comparison of differently synthesized gold-coated magnetic nanoparticles as 'dispersible electrodes', Electroanalysis 28(3) (2016) 431-438.

[62] E.T. Castellana, P.S. Cremer, Solid supported lipid bilayers: From biophysical studies to sensor design, Surf. Sci. Rep. 61(10) (2006) 429-444.

[63] M. Schulz, W.H. Binder, Mixed hybrid lipid/polymer vesicles as a novel membrane platform, Macromol. Rapid Commun. 36(23) (2015) 2031-2041.

[64] Liposomes: Methods and protocols, Volume 2: Biological membrane models, 1 ed., Humana Press, New York, NY, 2010.

[65] D. Xu, Q. Cheng, Surface-bound lipid vesicles encapsulating redox species for amperometric biosensing of pore-forming bacterial toxins, J. Am. Chem. Soc. 124(48) (2002) 14314-5.

[66] Q. Cheng, S. Zhu, J. Song, N. Zhang, Functional lipid microstructures immobilized on a gold electrode for voltammetric biosensing of cholera toxin, Analyst 129(4) (2004) 309-14.

[67] J. Vacek, M. Zatloukalova, J. Geleticova, M. Kubala, M. Modriansky, L. Fekete, J. Masek, F. Hubatka, J. Turanek, Electrochemical platform for the detection of transmembrane proteins reconsituted into liposomes, Anal. Chem. 88(8) (2016) 4548-4556. 
[68] J. Borch, F. Torta, S.G. Sligar, P. Roepstorff, Nanodiscs for immobilization of lipid bilayers and membrane receptors: Kinetic analysis of cholera toxin binding to a glycolipid receptor, Anal. Chem. 80(16) (2008) 6245-6252.

[69] V.A. Hernandez, K. Reijmar, K. Edwards, Label-free characterization of peptide-lipid interactions using immobilized lipodisks, Anal. Chem. 85(15) (2013) 7377-7384.

[70] A.L. Plant, Supported hybrid bilayer membranes as rugged cell membrane mimics, Langmuir 15(15) (1999) 5128-5135.

[71] Z. Wang, T. Wilkop, Q. Cheng, Characterization of micropatterned lipid membranes on a gold surface by surface plasmon resonance imaging and electrochemical signaling of a poreforming protein, Langmuir 21(23) (2005) 10292-6.

[72] T. Wilkop, D. Xu, Q. Cheng, Characterization of pore formation by streptolysin o on supported lipid membranes by impedance spectroscopy and surface plasmon resonance spectroscopy, Langmuir 23(3) (2007) 1403-9.

[73] T. Wilkop, D. Xu, Q. Cheng, Electrochemical characterization of pore formation by bacterial protein toxins on hybrid supported membranes, Langmuir 24(10) (2008) 5615-21. [74] E.C.M. Tse, C.J. Barile, J.P. Gewargis, Y. Li, S.C. Zimmerman, A.A. Gewirth, Anion transport through lipids in a hybrid bilayer membrane, Anal. Chem. 87(4) (2015) 2403-2409. [75] W. Ma, D.W. Li, T.C. Sutherland, Y. Li, Y.T. Long, H.Y. Chen, Reversible redox of NADH and $\mathrm{NAD}(+)$ at a hybrid lipid bilayer membrane using ubiquinone, J. Am. Chem. Soc. 133(32) (2011) 12366-12369.

[76] W. Ma, Y.L. Ying, L.X. Qin, Z. Gu, H. Zhou, D.W. Li, T.C. Sutherland, H.Y. Chen, Y.T. Long, Investigating electron-transfer processes using a biomimetic hybrid bilayer membrane system, Nat. Protoc. 8(3) (2013) 439-450.

[77] T.E. Wilkop, J. Sanborn, A.E. Oliver, J.M. Hanson, A.N. Parikh, On-demand self-assembly of supported membranes using sacrificial, anhydrobiotic sugar coats, J. Am. Chem. Soc. 136(1) (2014) 60-63.

[78] S.S. Hinman, C.J. Ruiz, G. Drakakaki, T.E. Wilkop, Q. Cheng, On-demand formation of supported lipid membrane arrays by trehalose-assisted vesicle delivery for SPR imaging, ACS Appl. Mater. Interfaces 7(31) (2015) 17122-30.

[79] M. Winterhalter, Black lipid membranes, Curr. Opin. Colloid Interface Sci. 5(3-4) (2000) 250-255. 
[80] B.A. Heitz, J.H. Xu, I.W. Jones, J.P. Keogh, T.J. Comi, H.K. Hall, C.A. Aspinwall, S.S. Saavedra, Polymerized planar suspended lipid bilayers for single ion channel recordings: Comparison of several dienoyl lipids, Langmuir 27(5) (2011) 1882-1890.

[81] L.K. Bright, C.A. Baker, R. Branstrom, S.S. Saavedra, C.A. Aspinwall, Methacrylate polymer scaffolding enhances the stability of suspended lipid bilayers for ion channel recordings and biosensor development, ACS Biomater. Sci. Eng. 1(10) (2015) 955-963.

[82] L.K. Bright, C.A. Baker, M.T. Agasid, L. Ma, C.A. Aspinwall, Decreased aperture surface energy enhances electrical, mechanical, and temporal stability of suspended lipid membranes, ACS Appl. Mater. Interfaces 5(22) (2013) 11918-11926.

[83] C.A. Baker, L.K. Bright, C.A. Aspinwall, Photolithographic fabrication of microapertures with well-defined, three-dimensional geometries for suspended lipid membrane studies, Anal. Chem. 85(19) (2013) 9078-9086.

[84] C.A. Baker, C.A. Aspinwall, Emerging trends in precision fabrication of microapertures to support suspended lipid membranes for sensors, sequencing, and beyond, Anal. Bioanal. Chem. 407(3) (2015) 647-652.

[85] J. Schmidt, Membrane platforms for biological nanopore sensing and sequencing, Curr. Opin. Biotechnol. 39 (2016) 17-27.

[86] R. Stefureac, Y.T. Long, H.B. Kraatz, P. Howard, J.S. Lee, Transport of alpha-helical peptides through alpha-hemolysin and aerolysin pores, Biochemistry 45(30) (2006) 9172-9179. [87] H.Y. Wang, Y. Li, L.X. Qin, A. Heyman, O. Shoseyov, I. Willner, Y.T. Long, H. Tian, Single-molecule DNA detection using a novel SP1 protein nanopore, Chem. Commun. 49(17) (2013) 1741-1743.

[88] C. Cao, Y.L. Ying, Z.L. Hu, D.F. Liao, H. Tian, Y.T. Long, Discrimination of oligonucleotides of different lengths with a wild-type aerolysin nanopore, Nat. Nanotechnol. (2016).

[89] Z. Gu, Y.L. Ying, C. Cao, P.G. He, Y.T. Long, Accurate data process for nanopore analysis, Anal. Chem. 87(2) (2015) 907-913.

[90] Y.L. Ying, C. Cao, Y.T. Long, Single molecule analysis by biological nanopore sensors, Analyst 139(16) (2014) 3826-3835. 
[91] H.Y. Wang, Y.L. Ying, Y. Li, H.B. Kraatz, Y.T. Long, Nanopore analysis of beta-amyloid peptide aggregation transition induced by small molecules, Anal. Chem. 83(5) (2011) 17461752.

[92] H.Y. Wang, Z. Gu, C. Cao, J. Wang, Y.T. Long, Analysis of a single alpha-synuclein fibrillation by the interaction with a protein nanopore, Anal. Chem. 85(17) (2013) 8254-8261.

[93] Y.L. Ying, H.Y. Wang, T.C. Sutherland, Y.T. Long, Monitoring of an ATP-binding aptamer and its conformational changes using an alpha-hemolysin nanopore, Small 7(1) (2011) 87-94. [94] Y.L. Ying, D.W. Li, Y. Li, J.S. Lee, Y.T. Long, Enhanced translocation of poly(dT)(45) through an alpha-hemolysin nanopore by binding with antibody, Chem. Commun. 47(20) (2011) 5690-5692.

[95] X. Zhang, J.J. Zhang, Y.L. Ying, H. Tian, Y.T. Long, Single molecule analysis of lightregulated RNA: Spiropyran interactions, Chem. Sci. 5(7) (2014) 2642-2646.

[96] A.B. Dahlin, B. Dielacher, P. Rajendran, K. Sugihara, T. Sannomiya, M. Zenobi-Wong, J. Voros, Electrochemical plasmonic sensors, Anal. Bioanal. Chem. 402(5) (2012) 1773-84.

[97] A. Abbas, M.J. Linman, Q. Cheng, New trends in instrumental design for surface plasmon resonance-based biosensors, Biosens. Bioelectron. 26(5) (2011) 1815-1824.

[98] M. Couture, S.S. Zhao, J.F. Masson, Modern surface plasmon resonance for bioanalytics and biophysics, Phys. Chem. Chem. Phys. 15(27) (2013) 11190-11216.

[99] S.H. Baek, A.W. Wark, H.J. Lee, Dual nanoparticle amplified surface plasmon resonance detection of thrombin at subattomolar concentrations, Anal. Chem. 86(19) (2014) 9824-9829. [100] R.J. Heaton, A.W. Peterson, R.M. Georgiadis, Electrostatic surface plasmon resonance: Direct electric field-induced hybridization and denaturation in monolayer nucleic acid films and label-free discrimination of base mismatches, Proc. Nat. Acad. Sci. USA 98(7) (2001) 37013704.

[101] D.D. Schlereth, Characterization of protein monolayers by surface plasmon resonance combined with cyclic voltammetry 'in situ', J. Electroanal. Chem. 464(2) (1999) 198-207. [102] S. Boussaad, J. Pean, N.J. Tao, High-resolution multiwavelength surface plasmon resonance spectroscopy for probing conformational and electronic changes in redox proteins, Anal. Chem. 72(1) (2000) 222-226.

[103] S.P. Wang, X.P. Huang, X.N. Shan, K.J. Foley, N.J. Tao, Electrochemical surface plasmon resonance: Basic formalism and experimental validation, Anal. Chem. 82(3) (2010) 935-941. 
[104] J. Lu, J. Li, Charge transfer kinetics from surface plasmon resonance voltammetry, Anal. Chem. 86(8) (2014) 3882-6.

[105] B.W. Davis, M.J. Linman, K.S. Linley, C.D. Hare, Q. Cheng, Unobstructed electron transfer on porous polyelectrolyte nanostructures and its characterization by electrochemical surface plasmon resonance, Electrochim. Acta 55(15) (2010) 4468-4474.

[106] S.E. Salamifar, R.Y. Lai, Application of electrochemical surface plasmon resonance spectroscopy for characterization of electrochemical DNA sensors, Colloids Surf., B 122 (2014) 835-839.

[107] K.S. Phillips, J.H. Han, Q. Cheng, Development of a "membrane cloaking" method for amperometric enzyme immunoassay and surface plasmon resonance analysis of proteins in serum samples, Anal. Chem. 79(3) (2007) 899-907.

[108] S. Patskovsky, V. Latendresse, A.M. Dallaire, L. Dore-Mathieu, M. Meunier, Combined surface plasmon resonance and impedance spectroscopy systems for biosensing, Analyst 139(3) (2014) 596-602.

[109] M. Manesse, V. Stambouli, R. Boukherroub, S. Szunerits, Electrochemical impedance spectroscopy and surface plasmon resonance studies of DNA hybridization on gold/SiO(x) interfaces, Analyst 133(8) (2008) 1097-1103.

[110] J. Lu, W. Wang, S. Wang, X. Shan, J. Li, N. Tao, Plasmonic-based electrochemical impedance spectroscopy: Application to molecular binding, Anal. Chem. 84(1) (2012) 327-33. [111] C. MacGriff, S. Wang, P. Wiktor, W. Wang, X. Shan, N. Tao, Charge-based detection of small molecules by plasmonic-based electrochemical impedance microscopy, Anal. Chem. 85(14) (2013) 6682-7.

[112] W. Liang, S. Wang, F. Festa, P. Wiktor, W. Wang, M. Magee, J. LaBaer, N. Tao, Measurement of small molecule binding kinetics on a protein microarray by plasmonic-based electrochemical impedance imaging, Anal. Chem. 86(19) (2014) 9860-5.

[113] A.M. Dallaire, S. Patskovsky, A. Vallee-Belisle, M. Meunier, Electrochemical plasmonic sensing system for highly selective multiplexed detection of biomolecules based on redox nanoswitches, Biosens. Bioelectron. 71 (2015) 75-81.

[114] J. Zhang, R. Li, F.L. Jiang, B. Zhou, Q.Y. Luo, Q.L.Y. Yu, X.L. Han, Y. Lin, H. He, Y. Liu, Y.L. Wang, An electrochemical and surface plasmon resonance study of adsorption actions of DNA by escherichia coli, Colloids Surf., B 117 (2014) 68-74. 
[115] C. Wu, F.U. Rehman, J. Li, J. Ye, Y. Zhang, M. Su, H. Jiang, X. Wang, Real-time evaluation of live cancer cells by an in situ surface plasmon resonance and electrochemical study, ACS Appl. Mater. Interfaces 7(44) (2015) 24848-54.

[116] D.G. Rackus, M.H. Shamsi, A.R. Wheeler, Electrochemistry, biosensors and microfluidics: A convergence of fields, Chem. Soc. Rev. 44(15) (2015) 5320-40.

[117] C.Y. Chen, S.S. Hinman, J. Duan, Q. Cheng, Nanoglassified, optically-active monolayer films of gold nanoparticles for in situ orthogonal detection by localized surface plasmon resonance and surface-assisted laser desorption/ionization-MS, Anal. Chem. 86(24) (2014) 11942-11945.

[118] S.S. Hinman, C.Y. Chen, J. Duan, Q. Cheng, Calcinated gold nanoparticle arrays for onchip, multiplexed and matrix-free mass spectrometric analysis of peptides and small molecules, Nanoscale 8(3) (2016) 1665-75. 


\section{FIGURES}

a

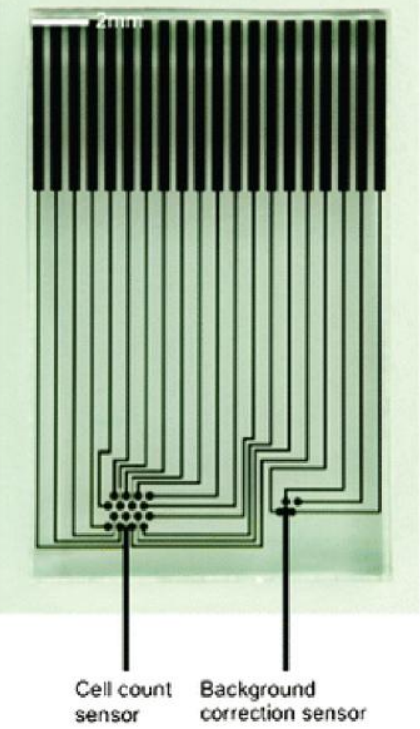

C

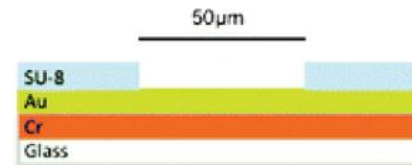

b
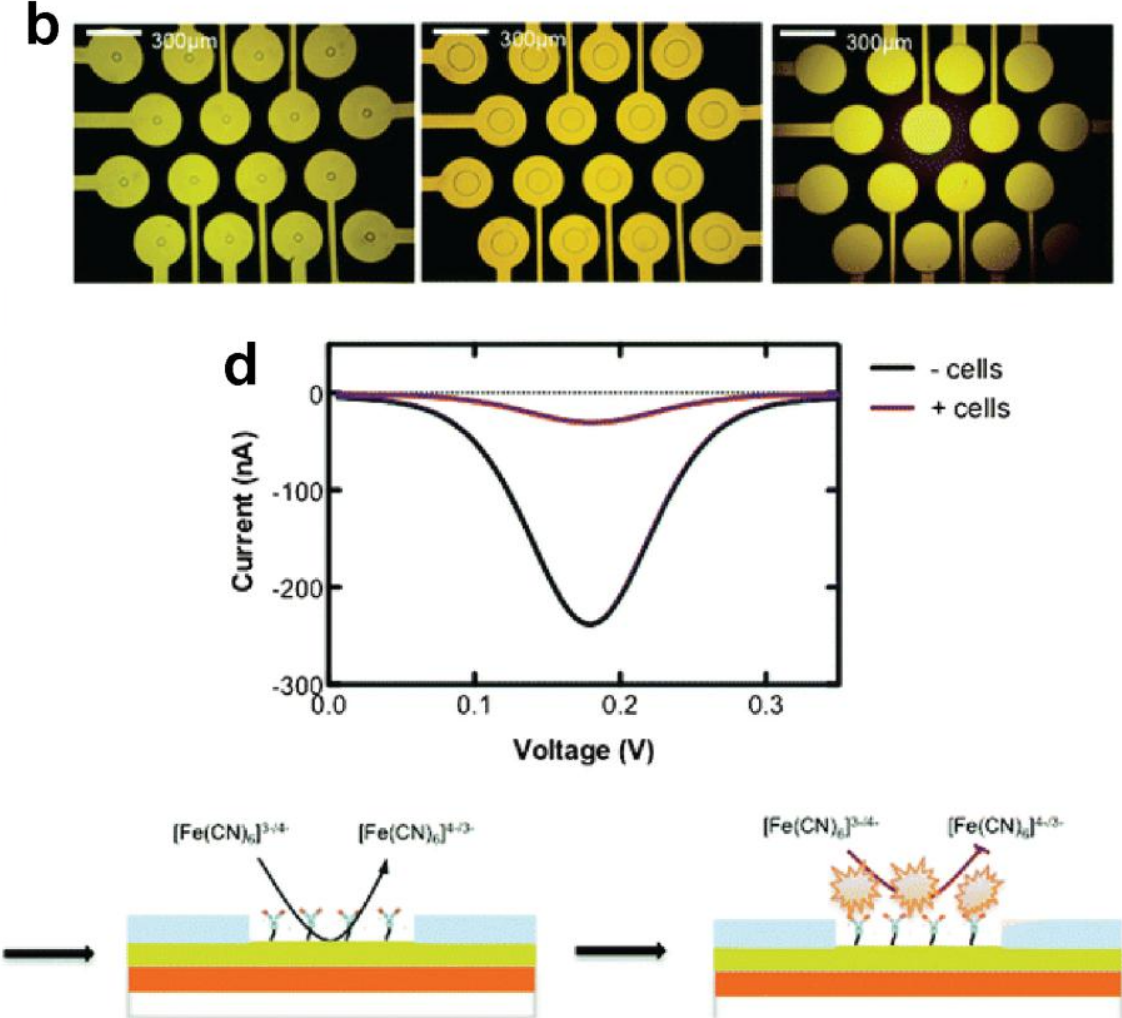

Figure 1. Electrochemical detection of prostate cancer cells with aperture sensor array. (a) Photograph of multiplexed chip. (b) Individual sensing areas of varying size. (c) Detection scheme utilizing redox reporter in solution. (d) Differential pulse voltammograms before and after binding of cells. Adapted from Ref. [9] with permission of The Royal Society of Chemistry. 


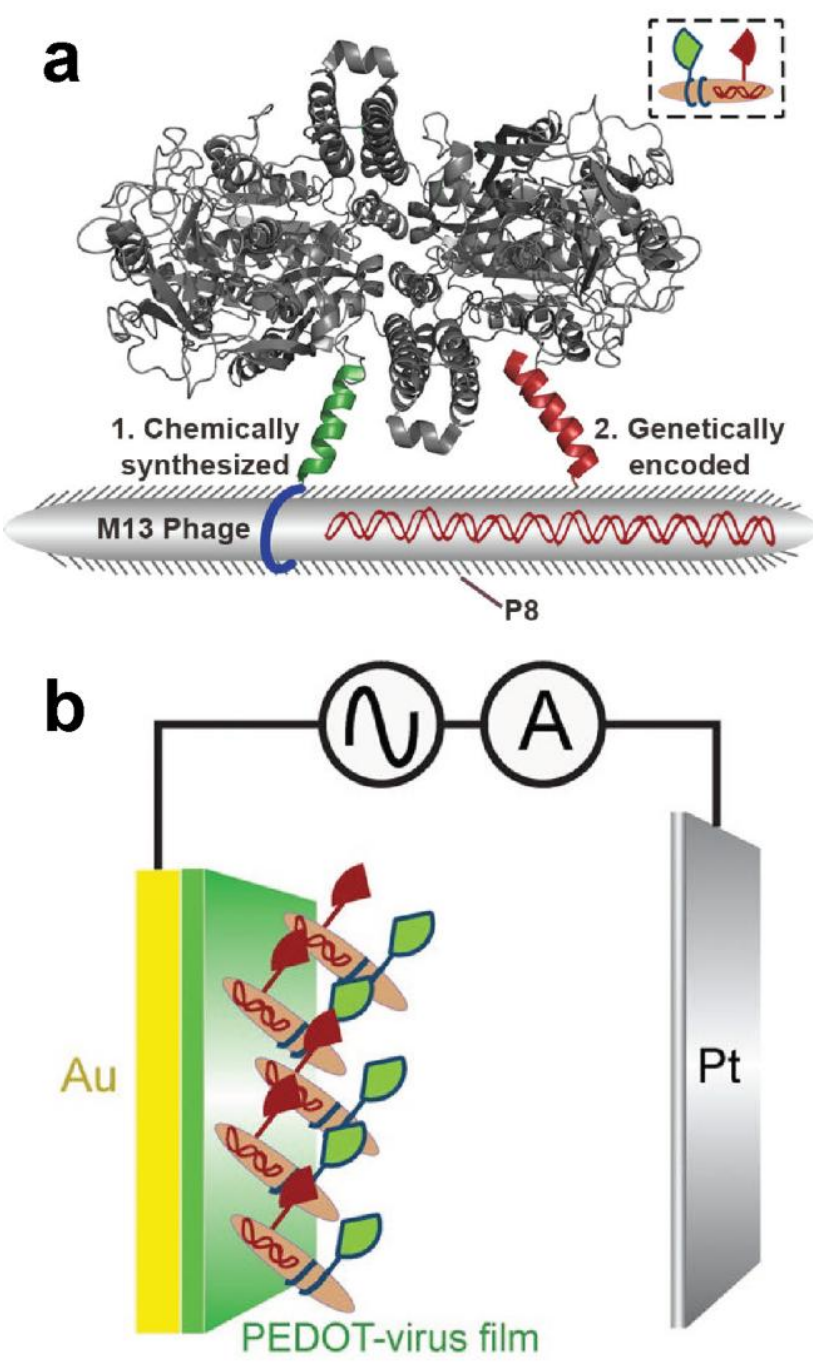

Figure 2. Dual ligand phage modification and sensing scheme. (a) Phage has one ligand genetically encoded, and the other synthetically derivitized for detection of prostate-specific membrane antigen. (b) Phage is co-assembled with PEDOT for electrochemical sensing. Adapted with permission from Ref. [24]. Copyright 2013 American Chemical Society. 
SLP-MB
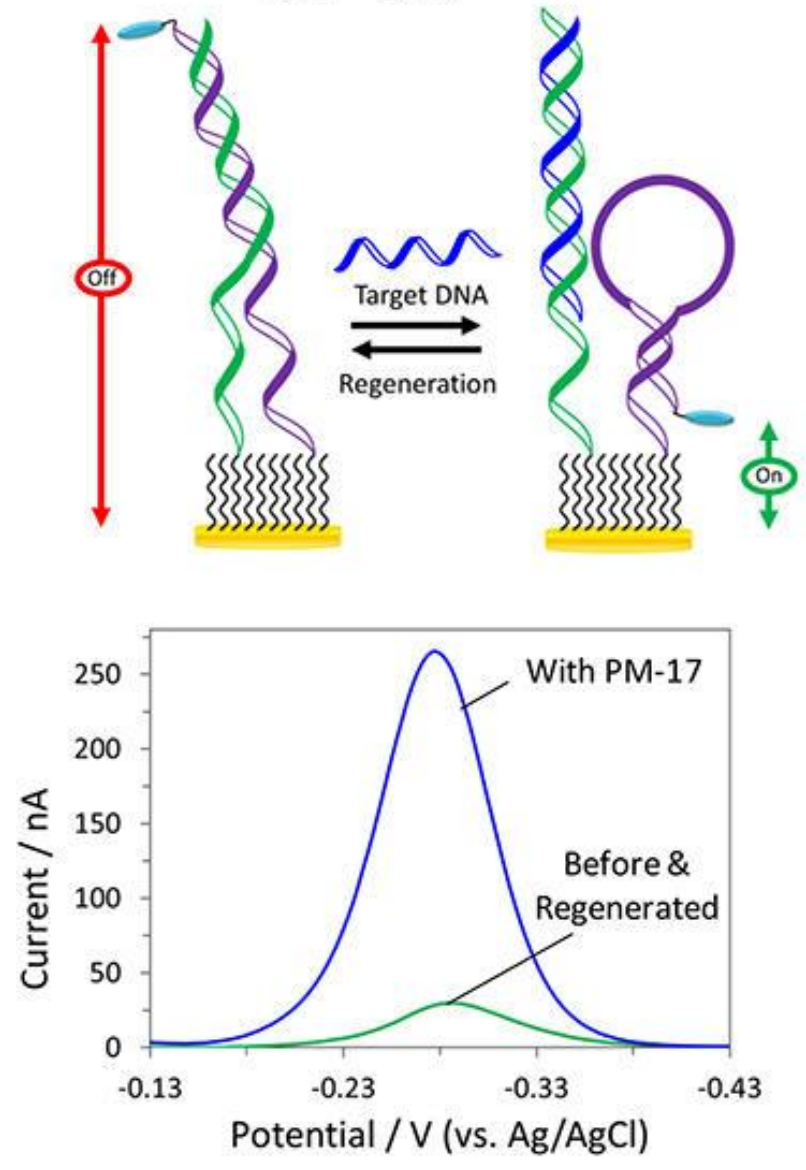

LB-MB
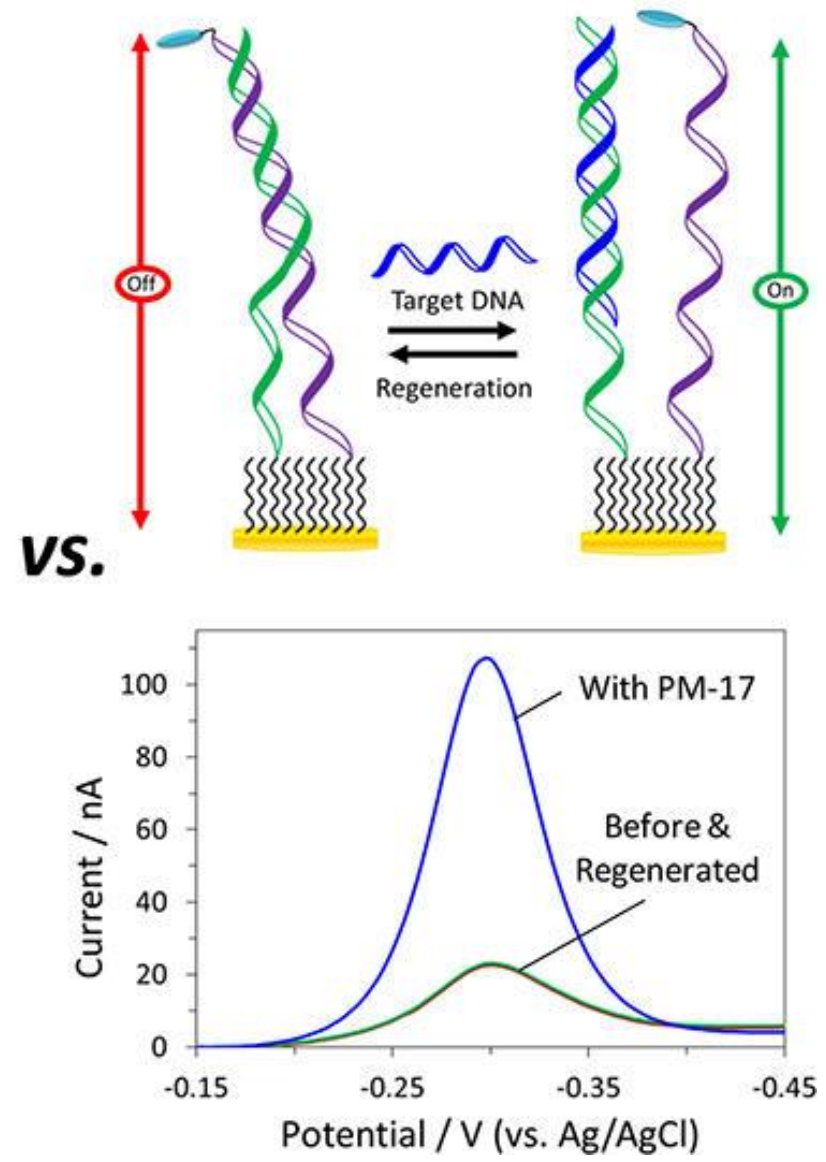

Figure 3. Hybridization induced conformational and dynamic DNA signal transduction mechanisms. The stem-loop methylene blue terminated probe (SLP-MB) is conformationally brought to the surface when displaced by the target ssDNA, while the linear methylene blue terminated probe (LB-MB) is dynamically brought to the surface when displaced by target ssDNA. Both restore the amperometric response. Reprinted with permission from Ref. [37]. Copyright 2013 American Chemical Society. 


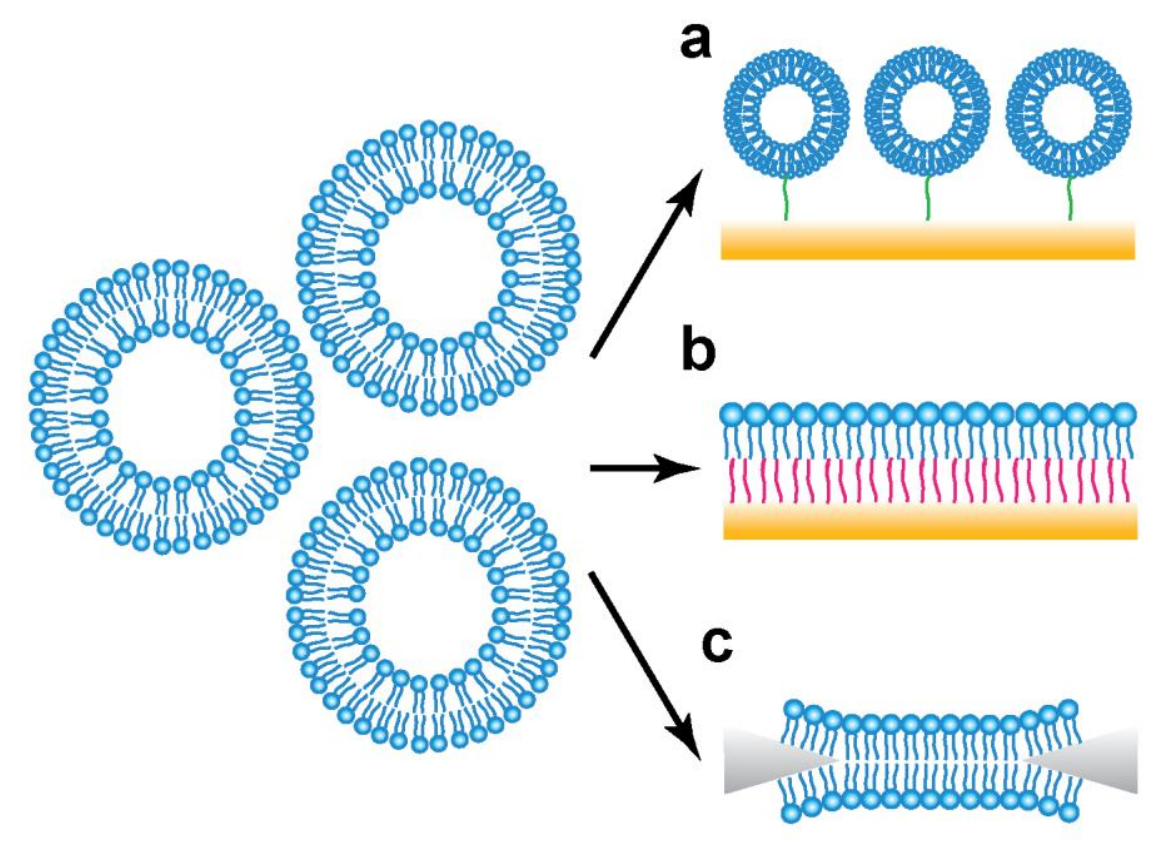

working electrode modified support

Figure 4. Lipid membrane assembly pathways for electrochemical measurements. (a) Tethered lipid vesicles or micelles from the working electrode. (b) Hybrid bilayer membrane, in which the lower leaflet is often a hydrophobic self- assembled monolayer. (c) Black lipid membrane, suspended within a narrow aperture.

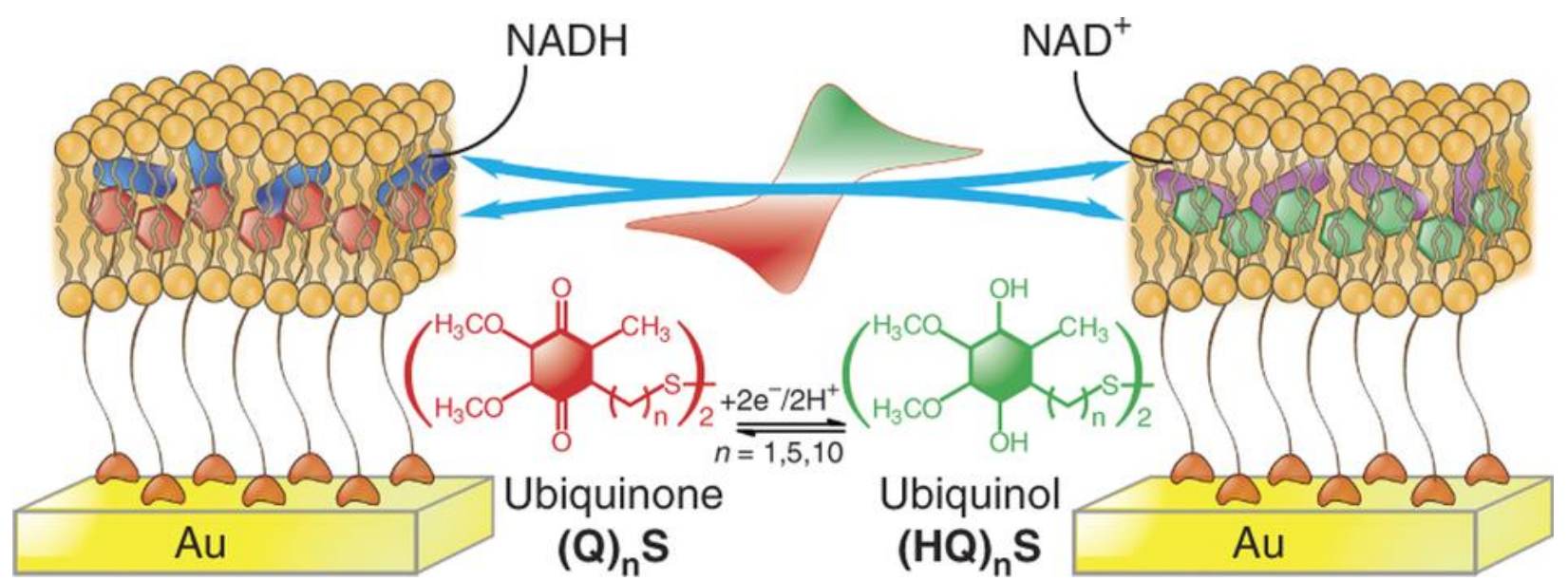

Figure 5. Tethered hybrid lipid bilayer incorporated with ubiquinone for monitoring the electrochemical processes of NADH/NAD ${ }^{+}$with cyclic voltammetry. Reprinted by permission from Macmillan Publishers Ltd: Nat. Protoc. Ref. [76], copyright 2013. 


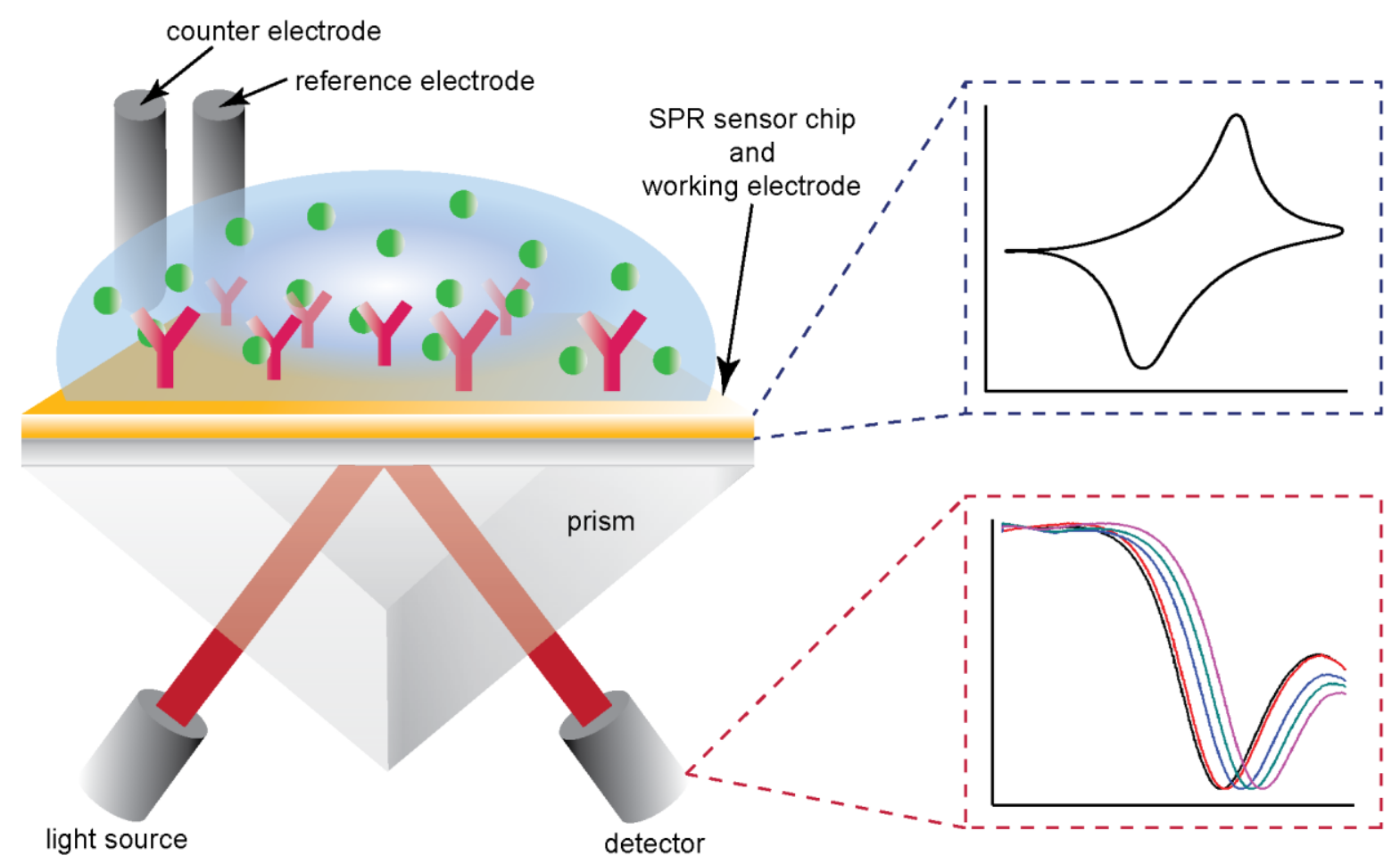

Figure 6. Surface plasmon resonance setup with integrated electrochemical equipment. The SPR sensor chip functions for reflectivity measurements, and as the working electrode. Counter and reference electrodes are generally placed in the flow medium. 

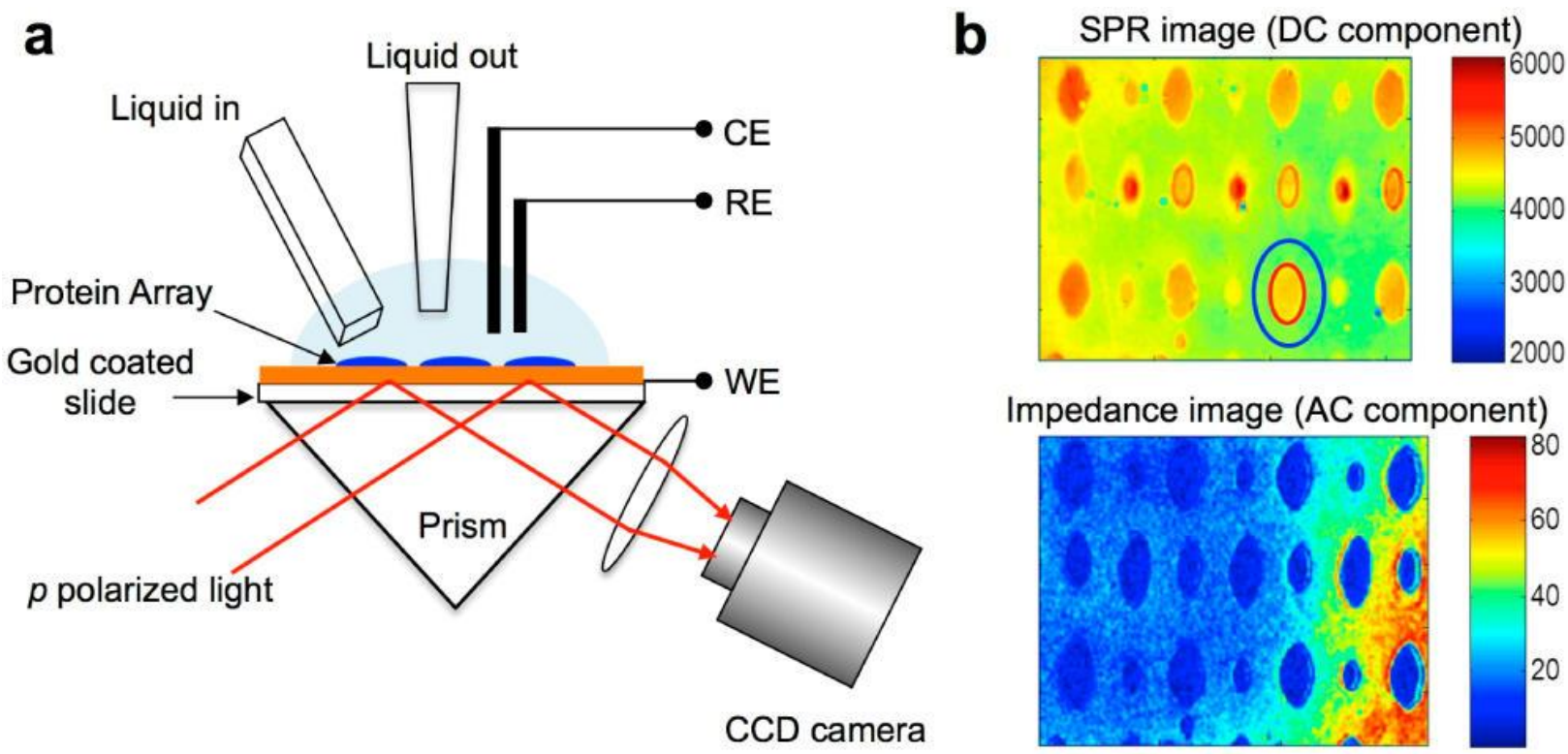

Impedance image (AC component)

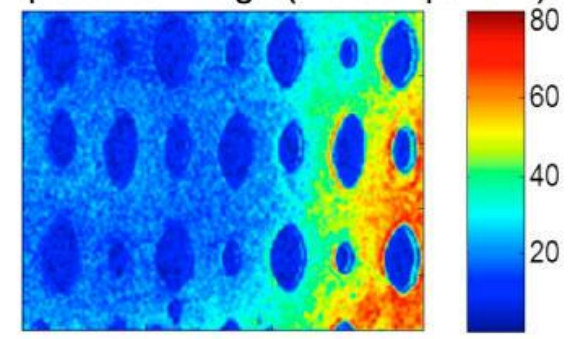

Figure 7. Working schematic of EIS-SPR and expected results. (a) Experimental setup for EISSPR imaging. Counter electrode and reference electrode are in the flow medium, while the working electrode is the SPR sensor chip. (b) Representative results, where the conventional SPR image is the DC component of the response, and the impedance can be imaged through the AC component. Reprinted with permission from Ref. [112]. Copyright 2014 American Chemical Society. 

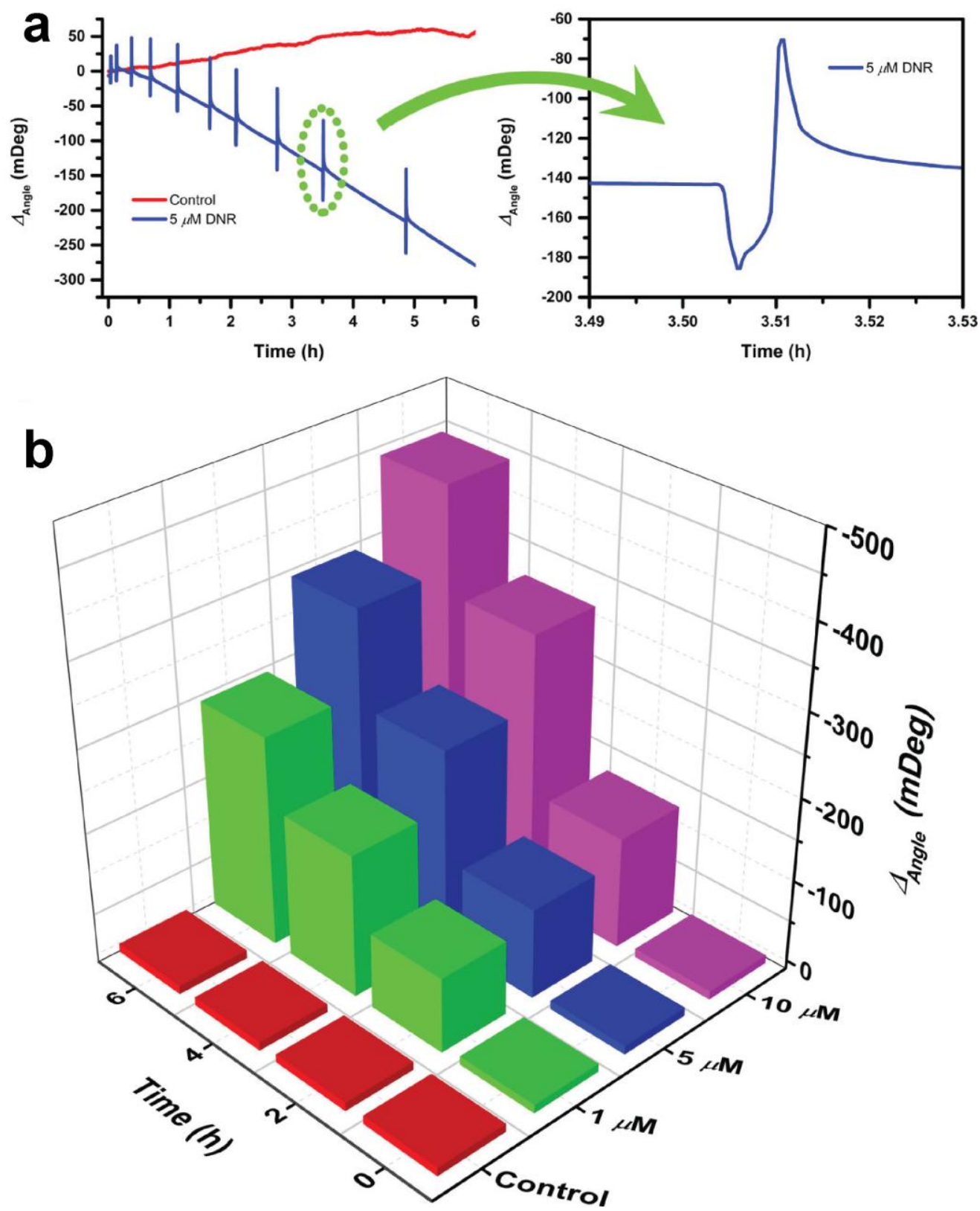

Figure 8. Results from EC-SPR studies of danorubicin exposures to HepG2 cells. (a) SPR sensorgrams, in which danorubicin exposure leads to decrease in signal from attached cells. Periodic spikes are the result of applied potentials from in situ CV experiments. (b) Collective data that takes exposure time and concentration of danorubicin into account. Adapted with permission from Ref. [115]. Copyright 2015 American Chemical Society. 


\section{GRAPHICAL ABSTRACT}

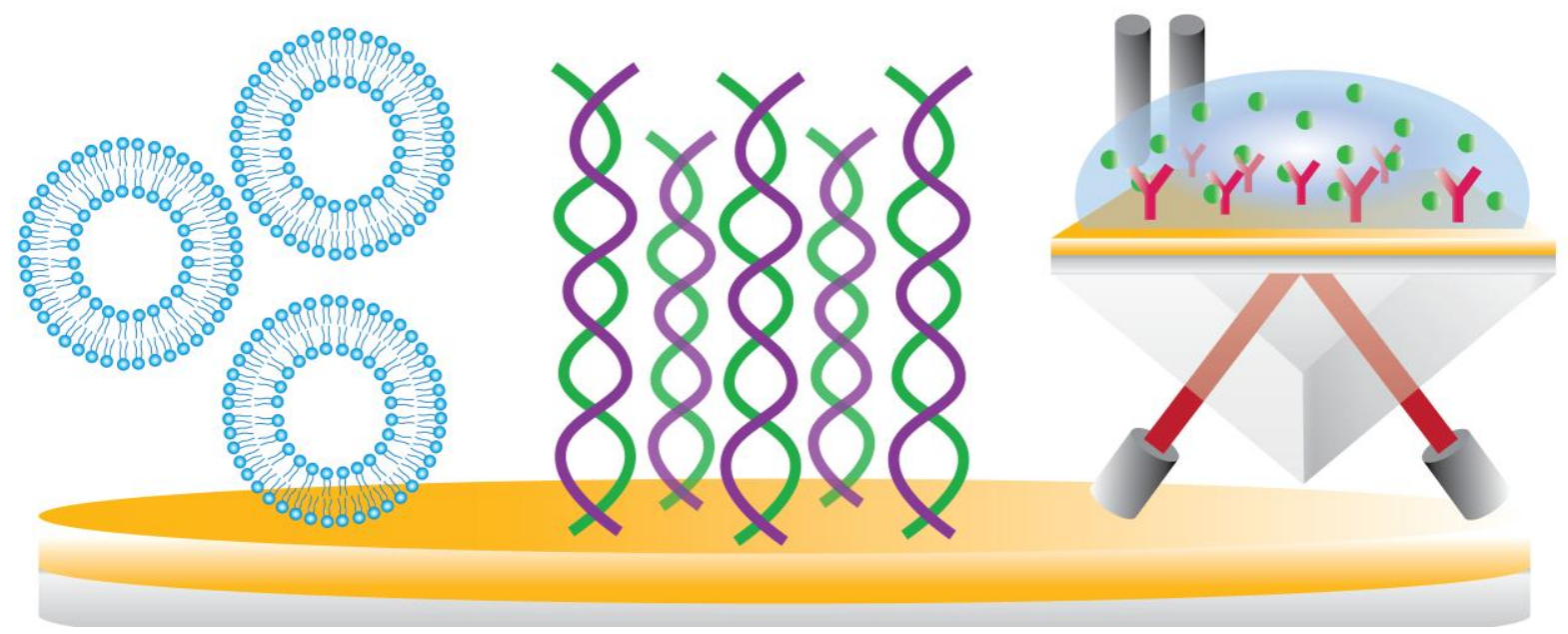

